\title{
Nanomechanical properties of molecular organic thin films
}

\author{
J. Caro and J. Fraxedas ${ }^{\text {a) }}$ \\ Institut de Ciència de Materials de Barcelona (CSIC), Campus de la UAB, E-08193, Bellaterra, Spain \\ P. Gorostiza and F. Sanz \\ Departament de Química-Física, Universitat de Barcelona, Martí i Franqués 1, E-08028, Barcelona, Spain
}

(Received 12 September 2000; accepted 5 March 2001)

\begin{abstract}
Using atomic force microscopy we have studied the nanomechanical response to nanoindentations of surfaces of highly oriented molecular organic thin films (thickness $\leqslant 1000 \mathrm{~nm}$ ). The Young's modulus $E$ can be estimated from the elastic deformation using Hertzian mechanics. For the quasi-one-dimensional metal tetrathiafulvalene tetracyanoquinodimethane $E \sim 20 \mathrm{GPa}$ and for the $\alpha$ phase of the $p$-nitrophenyl nitronyl nitroxide radical $E \sim 2 \mathrm{GPa}$. Above a few GPa, the surfaces deform plastically as evidenced by discrete discontinuities in the indentation curves associated to molecular layers being expelled by the penetrating tip. (c) 2001 American Vacuum Society.
\end{abstract}

[DOI: $10.1116 / 1.1368662]$

\section{INTRODUCTION}

The difficulty of synthesizing sufficiently large single crystals of molecular organic materials hinders the determination of several physical properties. Their mechanical properties, which are of interest when producing organicinorganic heterostructures, can be hardly characterized by conventional methods. However, estimations of parameters associated to the mechanical properties can be achieved by means of nanoindentation performed on thin films, which can provide larger areas of highly oriented and crystalline domains of the precursor materials. The study of the mechanical response of surfaces at the nanonewton force and nanometer penetration depth levels has been possible with atomic force microscopy $(\mathrm{AFM})^{1}$ and the interfacial force microscope $^{2}$ due to the higher force, lateral, and penetration depth resolution as compared to classical nanoindenters. However, the values of the parameters associated to the mechanical properties derived from the films may differ from those of the single crystals because of the presence of residual stress after growth, which calls for the characterization of their defect nature and density. This characterization can be achieved by imaging the perturbed surfaces after indentation.

In the present work we study the nanomechanical response of thin films of tetrathiafulvalene tetracyanoquinodimethane (TTF-TCNQ) and $p$-nitrophenyl nitronyl nitroxide ( $p$-NPNN) to nanoindentations performed with AFM. TTF-TCNQ is a charge transfer salt exhibiting a monoclinic crystal structure $\left(P 2_{1} / c, \quad a=12.298 \AA, \quad b=3.819 \AA, \quad c\right.$ $\left.=18.468 \AA, \beta=104.46^{\circ}\right)^{3}$ built up from parallel, segregated chains of donors (TTF) and acceptors (TCNQ). The electrical conductivity is highly anisotropic: the ratio of the $b$-axis conductivity to that along the $a$ axis is about $10^{3}$ at room temperature. $p$-NPNN was the first purely organic molecular material exhibiting bulk ferromagnetism. Four polymorphs are known for $p$-NPNN: $\alpha, \beta, \gamma$, and $\delta$. The orthorhombic $\beta$-phase $(\beta$ - $p$-NPNN:Fdd2, $a=10.960 \AA, b=19.350 \AA, c$

${ }^{\text {a) Electronic mail: fraxedas@icmab.es }}$
$=12.347 \AA)^{4}$ is the thermodynamically most stable one and undergoes a bulk ferromagnetic transition at $T_{c}=0.6 \mathrm{~K}$, while the metastable monoclinic $\alpha$ phase $(\alpha-p$-NPNN: $P 2{ }_{1} / c, \quad a=7.307 \AA, \quad b=7.596 \AA, \quad c=24.794 \AA, \quad \beta$ $\left.=93.543^{\circ 5}\right)$ is paramagnetic. ${ }^{6}$

\section{EXPERIMENT}

Thin films of TTF-TCNQ grown on ex situ cleaved $\mathrm{KCl}(001)$ substrates have been obtained by thermal sublimation in high vacuum $\left(\sim 10^{-6}\right.$ mbar $)$. The films consist of highly oriented and strongly textured rectangular-shaped microcrystals. The molecular (002) planes ( $a b$ planes) are parallel to the substrate surface and the microcrystals are oriented with their $a$ and $b$ axis parallel to both the [110] and [-110] substrate directions, respectively, due to the cubic symmetry of the substrates. ${ }^{7}$ The substrates were held at room temperature during the evaporation.

Thin films of $p$-NPNN obtained by thermal evaporation in high vacuum on glass slides and on ex situ cleaved $\mathrm{NaCl}(001)$ substrates held at room temperature crystallize in the monoclinic $\alpha$ phase. The molecular (002) planes (ab planes) are parallel to the substrate surface and exhibit a high degree of orientation but no in-plane texture. ${ }^{8}$

The nanoindentation and imaging were performed in ambient conditions with a Nanoscope III AFM (Digital Instruments, Santa Barbara, CA) using silicon cantilevers with spring constants $k \sim 3 \mathrm{Nm}^{-1}$ (nominal values $1-5 \mathrm{Nm}^{-1}$, supplied by Digital Instruments) and $k \sim 50 \mathrm{Nm}^{-1}$ (nominal values $38-74 \mathrm{Nm}^{-1}$, Nanosensors, Germany). After indentation the cantilever deflection $(\Delta)$ was calibrated using a clean glass slide and a diamond thin film for the $\sim 3$ and the $\sim 50$ $\mathrm{Nm}^{-1}$ cantilevers, respectively. Forces are evaluated by $F$ $=k \Delta$. The deformation or penetration $(\delta)$ is obtained by subtracting $\Delta$ from the $z$ displacement of the piezoscanner. The tip oscillation amplitude was reduced to zero while indenting with the $k \sim 3 \mathrm{Nm}^{-1}$ cantilever and for the $k \sim 50 \mathrm{Nm}^{-1}$ cantilever the approach was performed in contact mode in order to reduce noise. The sample to surface approach velocity $v$ is 


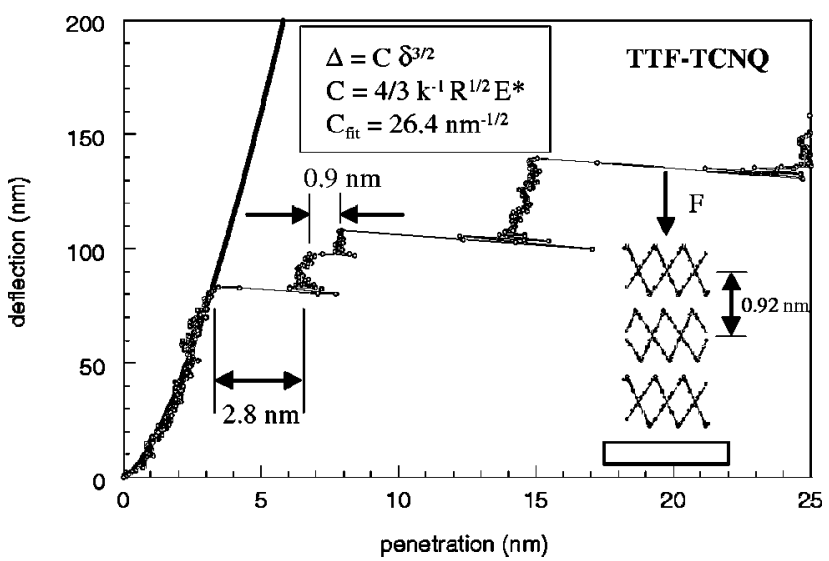

FIG. 1. Nanoindentation curve (deflection vs penetration) performed on a thin TTF-TCNQ film. The solid line represents the fit of the elastic region to the Hertzian model, which is indicated in the onset together with the value of the constant $C_{\text {fit }}$ obtained from the fit $\left(k \sim 3 \mathrm{Nm}^{-1}, v\right.$ $\sim 7.6 \mu \mathrm{m} \mathrm{s}^{-1}$ ). Also illustrated is the applied force $F$ perpendicular to the molecular $a b$ planes.

indicated in the figure captions. Imaging was performed with the AFM in tapping mode (TMAFM).

\section{RESULTS AND DISCUSSION}

\section{A. TTF-TCNQ}

Figure 1 shows a nanoindentation curve $(\Delta$ vs $\delta$ ) performed with a $\sim 3 \mathrm{Nm}^{-1}$ cantilever on a flat, defect-free region of a thin TTF-TCNQ film. These films are stable in air as evidenced by earlier TMAFM measurements. ${ }^{9}$ The applied force is perpendicular to the molecular $a b$ planes (see Fig. 1). The film behaves elastically below $F_{y} \sim 240 \mathrm{nN}$ $\left(=80 \mathrm{~nm} \times 3 \mathrm{Nm}^{-1}\right)$, where $F_{y}$ stands for the load at yield point. In this case the maximum elastic surface deformation $\delta_{y}$ is $\sim 3.5 \mathrm{~nm}$.

The shape of the loading curve in the elastic region may be modeled using Hertzian theory for a paraboloid indenting a plane. ${ }^{10,11}$ The Hertzian response is approximated by the expression $F=4 / 3 E^{*} R^{1 / 2} \delta^{3 / 2}$, where $E^{*}$ stands for the reduced Young's modulus defined as $1 / E^{*}=\left(1-\nu_{\text {tip }}^{2}\right) / E_{\text {tip }}$ $+\left(1-\nu^{2}\right) / E$. The Poisson's ratios of the tip and surface are represented by $\nu_{\text {tip }}$ and $\nu$, respectively, and the Young's moduli of the tip and surface by $E_{\text {tip }}$ and $E$, respectively. $R$ is the tip radius $(R \sim 7.5 \mathrm{~nm}$, nominal values $5-10 \mathrm{~nm})$. From the fit to the data we obtain $E^{*} \sim 22 \mathrm{GPa}$ and taking $E_{\text {tip }}$ $=130 \mathrm{GPa}, \nu_{\text {tip }}=0.28^{12}$ and $\nu \sim 0.4$, we obtain $E \sim 22 \mathrm{GPa}$. This estimation is of the order of previously reported values obtained on TTF-TCNQ single crystals, i.e., from temperature dependent measurements $(E \sim 10 \mathrm{GPa}),{ }^{13}$ from the slope of the acoustic branches measured by neutron scattering on single crystals $(E \sim 20 \mathrm{GPa}),{ }^{14}$ from determinations based on the sound velocity by ultrasonic techniques $(E \sim 34 \mathrm{GPa})^{15}$ and on the inverse of the compressibility coefficients $(E$ $\sim 55 \mathrm{GPa}) .{ }^{16}$ For comparison $E$ is typically less than $\sim 5$ $\mathrm{GPa}$ in the case of polymers. ${ }^{17}$

The mean applied stress normal to the surface at plastic yield $\sigma_{p}\left(=F_{y} / \pi R \delta_{y}\right)$ is $\sim 4 \mathrm{GPa}$ after averaging over sev-
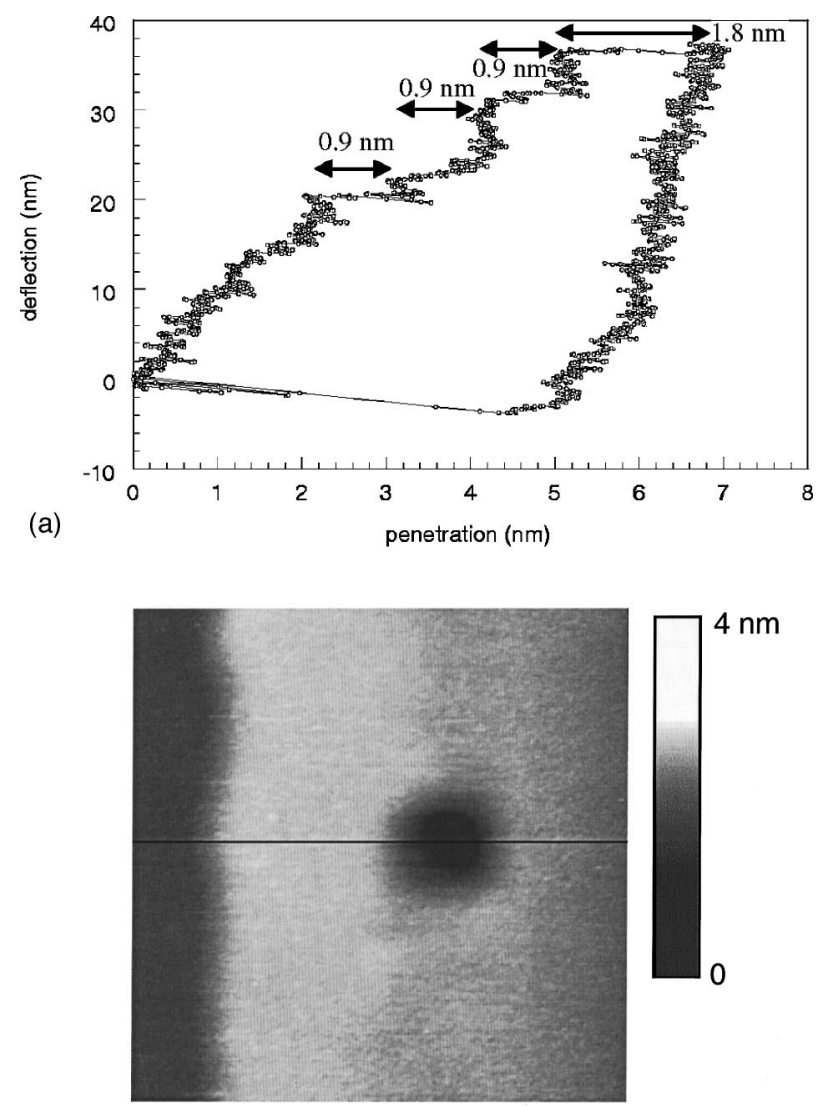

0

$60 \mathrm{~nm}$

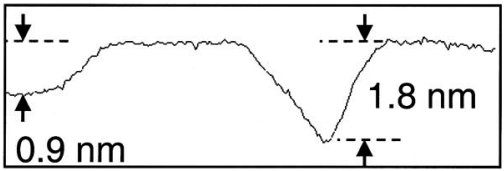

(b)

FIG. 2. (a) Nanoindentation curve (deflection vs penetration) performed on a thin TTF-TCNQ film $\left(k \sim 3 \mathrm{Nm}^{-1}, v \sim 2.0 \mu \mathrm{m} \mathrm{s}^{-1}\right)$. (b) TMAFM image of the indented region and corresponding line profile. The resultant plastic deformation can be compared to a monomolecular step.

eral indentation curves obtained with different tips. This value is comparable to, i.e., $\sigma_{p} \sim 7 \mathrm{GPa}$ for $\mathrm{Au}(111)^{18}$ and $\sigma_{p} \sim 10 \mathrm{GPa}$ for $\mathrm{MgO}(100) .{ }^{19}$ The estimated critical shear stress $\tau_{c}$ is $\sim 2 \mathrm{GPa}$ using $\tau_{c} \sim 0.5 \sigma_{p} \quad\left(\tau_{c}\right.$ is evaluated using Eqs. 209(k) and 218 from Ref. 10 for $\nu=0.4$ ). In the estimations we neglect anisotropy effects. ${ }^{18}$ Molecular organic materials are anisotropic and the films are layered (see, i.e., Fig. 1), with their most energetic planes (containing the shortest contacts) parallel to the substrate surface, thus implying that the interlayer interactions are rather weak (basically of van der Waals type). ${ }^{20}$

Above $\sigma_{p}$ the material deforms plastically as evidenced by discrete discontinuities (multiples of $\approx 0.9 \mathrm{~nm}$ ) associated to molecular layers being expelled by the penetrating tip (the distance between two consecutive $a b$-molecular planes is $0.92 \mathrm{~nm}$ ). This is illustrated in Fig. 1, where discontinuities of $\approx 0.9$ and $2.8 \mathrm{~nm}$ are observed just above the yield point. Figures 2(a) and 2(b) show a nanoindentation curve $(k$ 

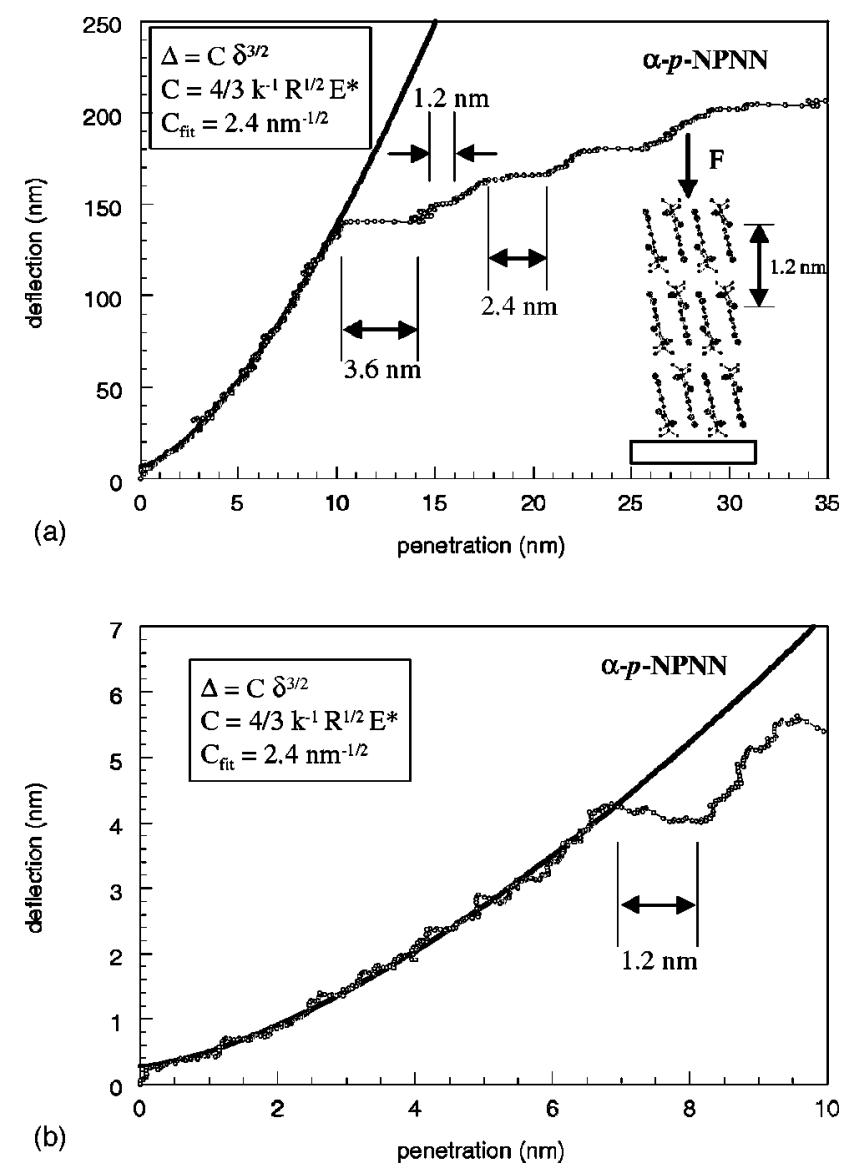

FIG. 3. Nanoindentation curves (deflection vs penetration) performed on an as-grown thin $\alpha$-p-NPNN film. The solid line represents the fit of the elastic region to the Hertzian model, which is indicated in the onset together with the value of the constant $C_{\text {fit }}$ obtained from the fit. (a) $k \sim 3 \mathrm{Nm}^{-1}, v$ $\sim 14.0 \mu \mathrm{m} \mathrm{s}^{-1}$, (b) $k \sim 50 \mathrm{Nm}^{-1}, v \sim 1.4 \mu \mathrm{m} \mathrm{s}^{-1}$. Also illustrated in (a) is that the applied force $F$ is perpendicular to the molecular $a b$ planes.

$\sim 3 \mathrm{Nm}^{-1}$ ) and a TMAFM image taken after the indentation, respectively. The plastic effect of the nanoindentation on the surface is clearly observed in Fig. 2(b). The total tip penetration $(\sim 5 \times 0.9 \mathrm{~nm})$ is larger than the measured depth of the induced defect $(\approx 1.8 \mathrm{~nm})$, which is twice the height of the close-lying step in Fig. 2(b) (step height $=0.92 \mathrm{~nm}$ ). When the tip penetrates, i.e., $\sim 3 \times 0.9 \mathrm{~nm}$ (not shown) the measured depth is $\approx 0.9 \mathrm{~nm}$. The fact that the measured depths systematically correspond nearly to either one or two molecular layers would suggest an ordered relaxation of the material upon unloading. The material expelled by the penetrating tip most probably evaporates during the subsequent scan process of image acquisition even if lateral forces are minimized, as is the case of the tapping scanning mode. ${ }^{21}$ No indentation-induced dislocations are observed in the vicinity of the indented region.

\section{B. $p$-NPNN}

Figure 3(a) shows a nanoindentation curve $\left(k \sim 3 \mathrm{Nm}^{-1}\right)$ performed on an as-grown $\alpha-p$-NPNN thin film. The applied force is perpendicular to the molecular $a b$ planes. The film behaves elastically below $F_{y} \sim 420 \mathrm{nN}\left(=140 \mathrm{~nm} \times 3 \mathrm{Nm}^{-1}\right)$ with $\delta_{y} \sim 10 \mathrm{~nm}$. A fit to the Hertzian model of the elastic response gives $E_{\alpha} \sim 1.7 \mathrm{GPa}$ (see discussion earlier). After averaging over several indentation curves obtained with different tips we obtain $\sigma_{p \alpha} \sim 1.3 \mathrm{GPa}$ and $\tau_{c} \sim 0.6 \mathrm{GPa}$. As for the TTF-TCNQ case, above the yield point discrete discontinuities corresponding to multiples of the distance between two consecutive $a b$ planes $(=1.2 \mathrm{~nm})$ are observed [see Fig. 3(a)]. A Hertzian fit has been also performed on the nanoindentation curve obtained with the $k \sim 50 \mathrm{Nm}^{-1}$ cantilevers [see Fig. 3(b)]. In this case $E_{\alpha} \sim 3.0 \mathrm{GPa}$ and $\sigma_{p \alpha}$ $\sim 1.4 \mathrm{GPa}$. Above the yield point the discontinuity is close to $1.2 \mathrm{~nm}$, although its determination is not as clear as for the $k \sim 3 \mathrm{Nm}^{-1}$ case shown in Fig. 3(a).

The nanometer-scale surface morphology of the $\alpha$ - $p$-NPNN thin films is rather complex. It is composed by a random distribution of dislocations (spirals) of opposite sign interacting in pairs (Frank-Read mechanism of growth), each spiral emerging from a hollow core. ${ }^{22}$ The relative small distance $L$ between emerging points of the interacting spirals $(L>50 \mathrm{~nm})$ indicates a rather high dislocation density, caused by a rapid in-plane crystallization from an initially amorphous state. ${ }^{23}$ The $\alpha$ phase transforms spontaneously to the more stable $\beta$ phase. However, this transformation is inhibited if the thickness of the as-grown $\alpha$-phase films lies below a critical thickness $(\sim 1 \mu \mathrm{m})$. The stabilization is induced by residual stress after growth. ${ }^{24}$ The accumulated stress field after growth induces an increase of the activation energy of formation of critical nuclei of the $\beta$ phase in the $\alpha$-phase matrix and thus enables the inhibition of this solid-solid phase transition. An estimate of the residual stress $\tau_{r}$ for thin films of $\alpha$-p-NPNN (thickness $\approx 1 \mu \mathrm{m})$ gives $^{22,25} \tau_{r} \sim 2 G b L^{-1}<0.02 \mathrm{GPa}$, where $b$ represents the Burgers vector of the dislocation $(b=1.2 \mathrm{~nm})$ and $G(\sim 0.6 \mathrm{GPa})$ the bulk modulus, approximated by the expression $G=E / 2(1+\nu)$, valid for isotropic materials. $\tau_{r}$, which is correlated to $\tau_{c},{ }^{26}$ is only roughly approximated because of the uncertainties associated to most of the parameters involved.

The estimated values of $E_{\alpha}, \sigma_{p \alpha}$, and $\tau_{c}$ for thin films may differ from their intrinsic values (single crystals), unavailable to our knowledge, because of the influence of residual stress after growth, since defects hinder the propagation of dislocations. This might be modeled, i.e., by the Hall-Petch equation, $\sigma_{p} \approx \sigma_{p}^{\text {int }}+A L^{-1 / 2}$, valid for grain boundaries, where $\sigma_{p}^{\text {int }}$ and $A$ represent the intrinsic stress (single crystal) normal to the surface at plastic yield and a constant, respectively, and where the grain size has been replaced by the distance between dislocations. ${ }^{27}$ Thermal stress is negligible because the substrates are held at room temperature during growth. Above $\sim 1 \mu \mathrm{m}$ in thickness the films transform to the $\beta$ phase. In this case the films are polycrystalline exhibiting two main crystallographic directions, with their (040) and (220) planes parallel to the surface. Unfortunately, it is not possible to differentiate with the AFM both orientations. An indentation curve $\left(k \sim 3 \mathrm{Nm}^{-1}\right)$ performed on flat regions of a transformed sample to the $\beta$ phase is shown in Fig. 4 [the force is applied perpendicularly either to 


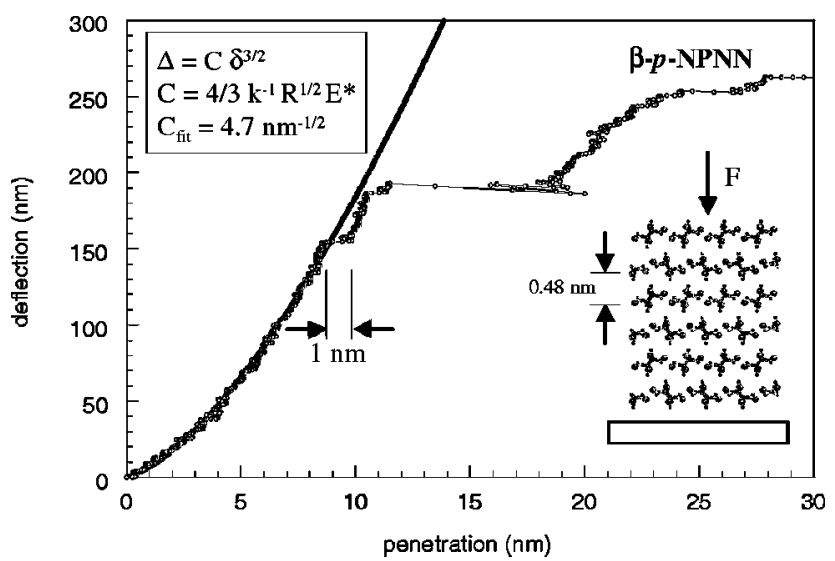

FIG. 4. Nanoindentation curve (deflection vs penetration) performed on a transformed thin $\beta$ - $p$-NPNN film $\left(k \sim 3 \mathrm{Nm}^{-1}, v \sim 15.3 \mu \mathrm{m} \mathrm{s}^{-1}\right)$. The solid line represents the fit of the elastic region to the Hertzian model, which is indicated in the onset together with the value of the constant $C_{\text {fit }}$ obtained from the fit. Also illustrated is the direction of the applied force with regard to the molecular distribution in the film (i.e., $F$ is perpendicular to the molecular $a c$ planes).

the (040) or (220) planes]. In this case the microcrystals exhibit terraces with a mean step separation of $\sim 100 \mathrm{~nm}^{24}$ From the Hertzian fit we obtain $E_{\beta} \sim 3.3 \mathrm{GPa}$ and $\sigma_{p \beta}$ $\sim 2.1 \mathrm{GPa}$ after averaging over several indentation curves performed close and away from the steps. ${ }^{28} \mathrm{We}$ believe this estimation of the mechanical properties of the $\beta$ phase should be closer to the single crystal values because of strain relief of the structure upon transformation. Above the yield point a discrete discontinuity of $\sim 1 \mathrm{~nm}$ is observed, which corresponds to two times either the distance between two consecutive (040) or (220) planes. $E_{\beta}$ is always 2-3 times larger than $E_{\alpha}$, obtained from systematic measurements performed with the same cantilever, thus reducing the uncertainty associated to $k$ and $R$. We can thus conclude that $E_{\beta}^{\text {int }}$ $>E_{\alpha}^{\text {int }}$ and $\sigma_{\beta}^{\text {int }}>\sigma_{\alpha}^{\text {int }}$, because the density of dislocations upon transformation from the $\alpha$ to the $\beta$ phase is reduced. $E^{\text {int }}$ stands for the intrinsic values (single crystals) of the Young's moduli.

\section{SUMMARY}

We have characterized the mechanical response (elastic and plastic) to nanoindentations with AFM of thin films of two molecular organic materials exhibiting interesting physical properties: TTF-TCNQ and $p$-NPNN. The elastic response enables the estimation of parameters such as the Young's modulus $E$ and the yield point of plastic deformation determines the critical stress $\sigma_{p}$. In our case we obtain $E \sim 1-20 \mathrm{GPa}$ and $\sigma_{p} \sim 1-4 \mathrm{GPa}$. These mechanically soft materials are thus stronger than most polymers. Two polymorphs of $p$-NPNN, the metastable $\alpha$ (stabilized as thin film) and the thermodynamically most stable $\beta$ phases, have been compared. In this case $E_{\beta}>E_{\alpha}$.
Surface imaging before and after indentation gives valuable information concerning the nature and density of dislocations existing prior to indentation and those induced by indentation, which is relevant for thin films.

${ }^{1}$ G. M. Pharr and W. C. Oliver, MRS Bull. XVII, 28 (1992); N. A. Burnham and R. J. Colton, J. Vac. Sci. Technol. A 7, 2906 (1989).

${ }^{2}$ S. A. Joyce, R. C. Thomas, J. E. Houston, T. A. Michalske, and R. M. Crooks, Phys. Rev. Lett. 68, 2790 (1992).

${ }^{3}$ T. J. Kistenmacher, T. E. Philips, and D. O. Cowan, Acta Crystallogr., Sect. B: Struct. Crystallogr. Cryst. Chem. 30, 763 (1974).

${ }^{4}$ K. Agawa, T. Inabe, U. Nagashima, and Y. Maruyama, J. Chem. Soc. Chem. Commun. 520 (1990)

${ }^{5}$ P. M. Allemand, C. Fite, G. Srdanov, N. Keder, F. Wudl, and P. Canfield, Synth. Met. 41-43, 3291 (1991).

${ }^{6}$ M. Kinoshita, Jpn. J. Appl. Phys., Part 1 33, 5718 (1994).

${ }^{7}$ J. Fraxedas, J. Caro, A. Figueras, P. Gorostiza, and F. Sanz, J. Vac. Sci. Technol. A 16, 2517 (1998).

${ }^{8}$ J. Caro, J. Fraxedas, O. Jürgens, J. Santiso, C. Rovira, J. Veciana, and A. Figueras, Adv. Mater. 10, 608 (1998).

${ }^{9}$ J. Fraxedas, J. Caro, A. Figueras, P. Gorostiza, and F. Sanz, Surf. Sci. 395, 205 (1998).

${ }^{10} \mathrm{~S}$. Timoshenko and J. N. Goodier, Theory of Elasticity, 2nd ed. (McGrawHill, New York, 1951), Vol. 1, p. 372.

${ }^{11}$ N. A. Burnham, R. J. Colton, and H. M. Pollock, Nanotechnology 4, 64 (1993); N. A. Burnham et al., ibid. 8, 67 (1997).

${ }^{12}$ Landolt-Börnstein, Numerical Data and Functional Relationships in Science and Technology, Group III: Crystal and Solid State Physics, 1st ed. edited by O. Madelung (Springer, Berlin, 1982), Vol. 17.

${ }^{13}$ J. C. Phillips, J. Solid State Chem. 20, 211 (1977).

${ }^{14}$ J. P. Pouget, S. M. Shapiro, G. Shirane, A. F. Garito, and A. J. Heeger, Phys. Rev. B 19, 1792 (1979).

${ }^{15}$ T. Tiedje, R. R. Haering, M. N. Jericho, W. A. Roger, and A. Simpson, Solid State Commun. 23, 713 (1977)

${ }^{16}$ A. Filhol, G. Bravic, J. Gaultier, D. Chasseau, and C. Vettier, Acta Crystallogr., Sect. B: Struct. Crystallogr. Cryst. Chem. 37, 1225 (1981).

${ }^{17}$ A. L. Weisenhorn, M. Khorsandi, S. Kasas, V. Gotzos, and H.-J. Butt, Nanotechnology 4, 106 (1993); J. P. Aimé, Z. Elkaakour, C. Odin, T. Bouhacina, D. Michel, J. Curély, and A. Dautant, J. Appl. Phys. 76, 754 (1994); A. J. Howard, R. R. Rye, and J. E. Houston, ibid. 79, 1885 (1996); T. Kajiyama, K. Tanaka, S.-R. Ge, and A. Takahara, Prog. Surf. Sci. 52, 1 (1996)

${ }^{18}$ J. D. Kiely and J. E. Houston, Phys. Rev. B 57, 12588 (1998).

${ }^{19}$ P. F. M. Terán Arce, G. Andreu Riera, P. Gorostiza, and F. Sanz, Appl. Phys. Lett. 77, 839 (2000)

${ }^{20}$ S. Molas, J. Caro, J. Santiso, A. Figueras, J. Fraxedas, M. Fourmigué, C. Mézière and P. Batail, J. Cryst. Growth 218, 399 (2000).

${ }^{21}$ H. P. Lang, B. Erler, A. Rossberg, M. Piechotka, E. Kaldis, and H.-J. Güntherodt, J. Vac. Sci. Technol. B 14, 970 (1996).

${ }^{22}$ J. Fraxedas, J. Caro, A. Figueras, P. Gorostiza, and F. Sanz, Surf. Sci. 415, 241 (1998).

${ }^{23}$ J. Caro, J. Fraxedas, and A. Figueras, J. Cryst. Growth 209, 146 (2000).

${ }^{24}$ J. Fraxedas, J. Caro, J. Santiso, A. Figueras, P. Gorostiza, and F. Sanz, Europhys. Lett. 48, 461 (1999).

${ }^{25}$ A. P. Sutton and R. W. Baluffi, Interfaces in Crystalline Materials, 2nd ed. (Clarendon, Oxford, 1995), Vol. 1, p. 547.

${ }^{26}$ J. E. Houston, Mater. Res. Soc. Symp. Proc. 440, 177 (1997).

${ }^{27}$ M. Ohring, The Materials Science of Thin Films, 1st ed. (Academic, San Diego, 1992), Vol. 1, Chap. 9, p. 409.

${ }^{28}$ J. D. Kiely, R. Q. Hwang, and J. E. Houston, Phys. Rev. Lett. 81, 4424 (1998). 